(c) 2011 IEEE. Personal use of this material is permitted. Permission from IEEE must be obtained for all other uses, in any current or future media, including reprinting/republishing this material for advertising or promotional purposes, creating new collective works, for resale or redistribution to servers or lists, or reuse of any copyrighted component of this work in other works. 


\section{On the Matter}

of

Classifying $\mathcal{N o d e}$ Connections

Colin J. Armstrong

Curtin University

Perth, Western Australia 
On the Matter of Classifying Node Connections

\title{
Bertrand Russell
}

$18^{\text {th }}$ May $1872-2^{\text {nd }}$ February 1970

\author{
The Odour of Petroleum
}


It is easier to see the "Things" than it is to see the "Links" between them

\section{EMOTIONS MAPPED BY NEW GEOGRAPHY}

Charts scek to Portray the. Psychological Currents of. Human Relationships.

\section{New York Times}

April 3, 1933

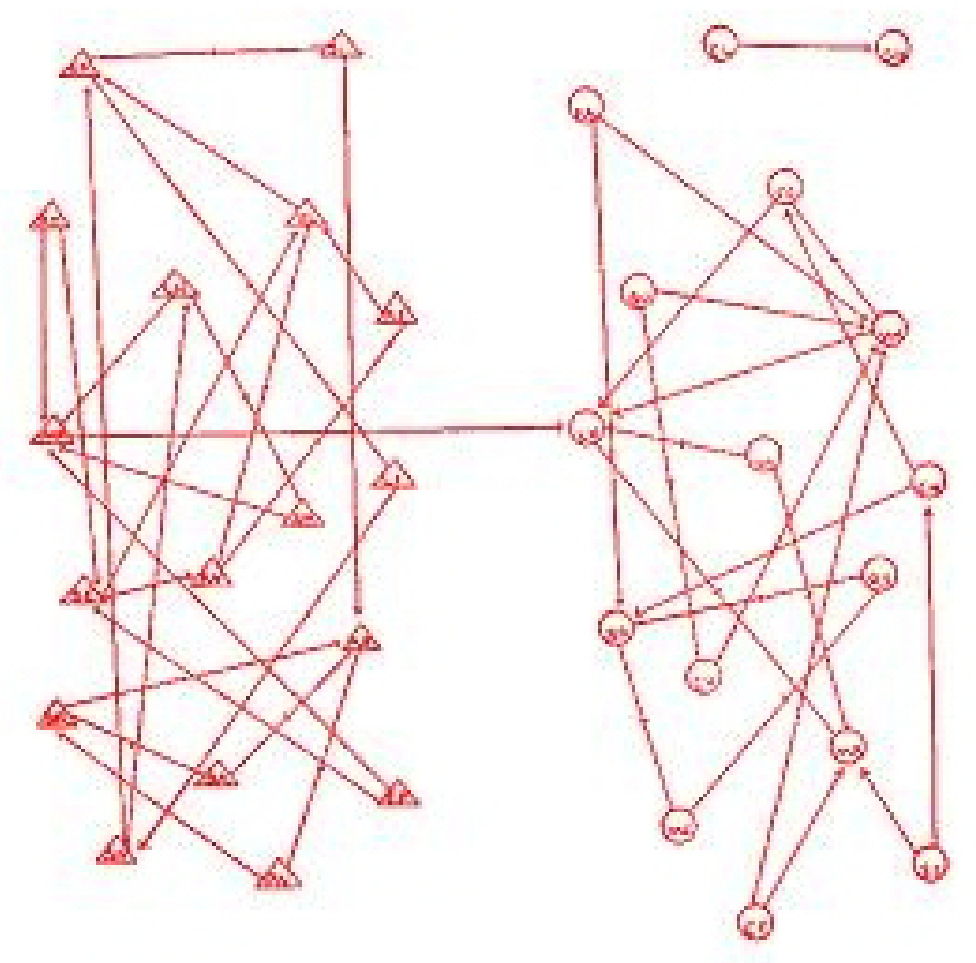

$\&$, there is no value in me explaining networks to you... but 


\section{Challenged by the Links - the Relationships}

\section{Traditional MAN Directionality}

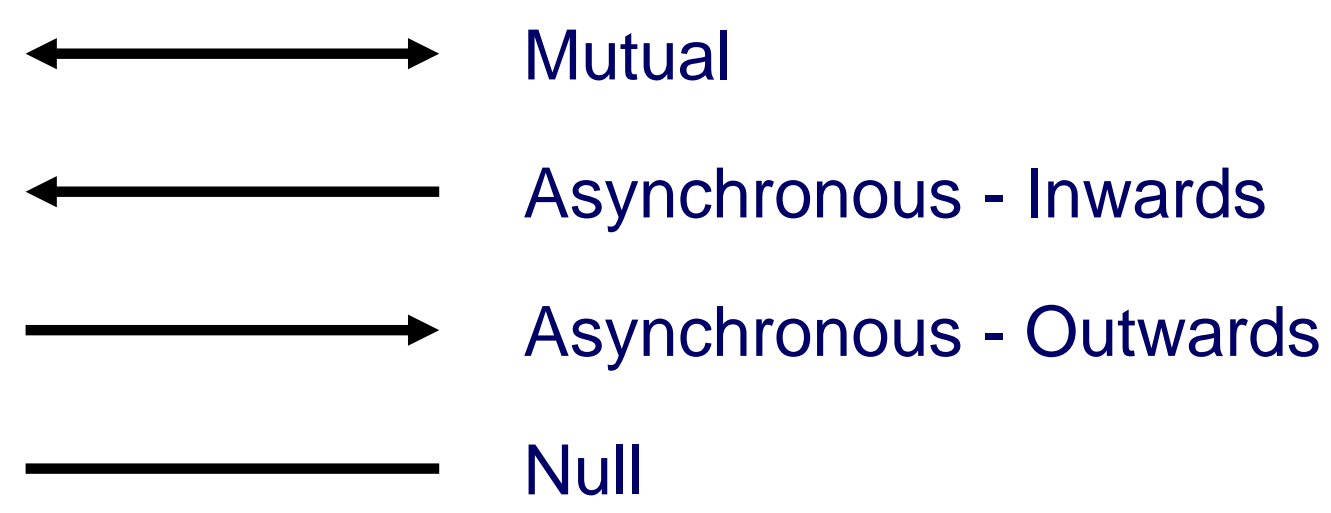

Started with a need to differentiate between a Node's 'Significant' and 'Insignificant' Null Relationships 


\section{And then...}

When networking forensic evidence, not only is the traditional MAN system a challenge...

But also the basic definition of networks and hubs within networks...

Something seems to be missing

So...

Back to basics and the drawing board... 


\section{Easy, and Simple}

\begin{tabular}{|c|c|c|c|c|c|}
\hline \multirow{3}{*}{ Node } & \multicolumn{2}{|c|}{ Link Type } & \multirow{3}{*}{ Nomenclature } & \multirow{3}{*}{$\begin{array}{l}\text { Binary } \\
\text { Value }\end{array}$} & \multirow{3}{*}{$\begin{array}{c}\text { Cell } \\
\text { Value }\end{array}$} \\
\hline & $Y$ & $\mathbf{N}$ & & & \\
\hline & 2 & 1 & & & \\
\hline 1 & 0 & 0 & Isolate & 00 & 0 \\
\hline 2 & 0 & 1 & oN & 01 & 1 \\
\hline 3 & 1 & 0 & Mo & 10 & 2 \\
\hline
\end{tabular}

Serves a basic purpose

Basic Nomenclature

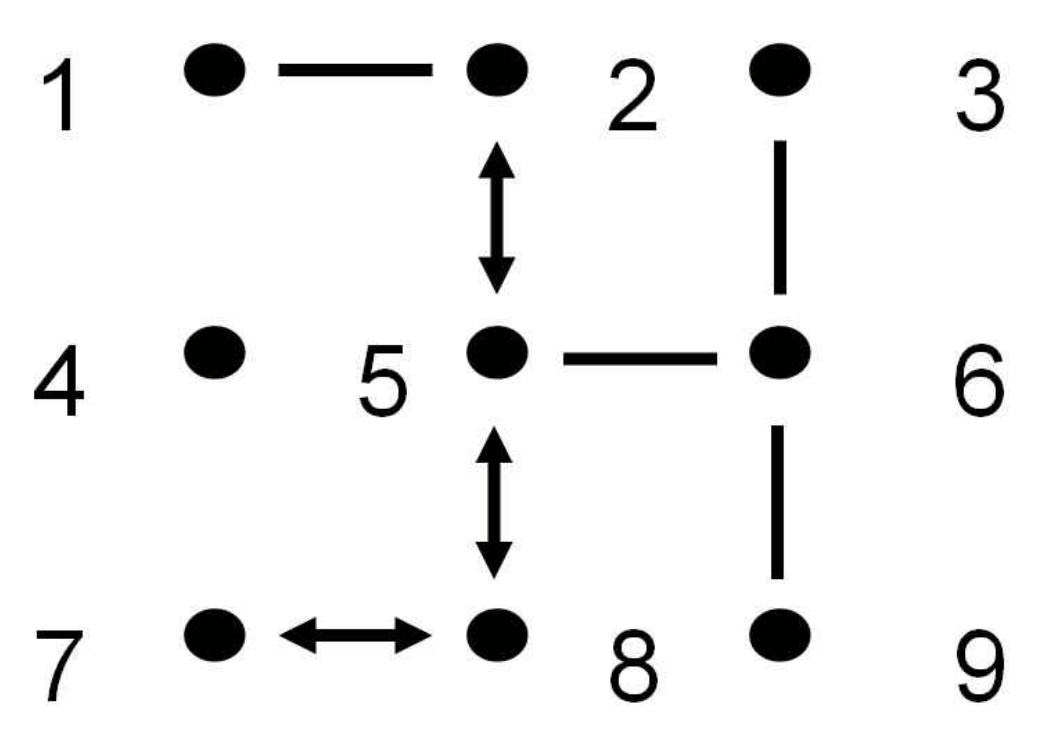




\section{Not so Easy, and Not so Simple}

\begin{tabular}{|c|c|c|c|c|c|c|c|}
\hline \multirow{3}{*}{ Node } & \multicolumn{4}{|c|}{ Link Type } & \multirow{3}{*}{ Nomenclature } & \multirow{3}{*}{ Code } & \multirow{3}{*}{$\begin{array}{l}\text { Cell } \\
\text { Value }\end{array}$} \\
\hline & $M$ & $\mathbf{A} \mathbf{i}$ & Ao & $\mathrm{N}$ & & & \\
\hline & 8 & 4 & 2 & 1 & & & \\
\hline 1 & 0 & 0 & 0 & 0 & Isolate & 0000 & 0 \\
\hline 2 & 0 & 0 & 0 & 1 & OOON & 0001 & 1 \\
\hline 3 & 0 & 0 & 1 & 0 & $\circ \circ A \circ \circ$ & 0010 & 2 \\
\hline 4 & 0 & 0 & 1 & 1 & $\circ \circ A^{\circ} \mathrm{N}$ & 0011 & 3 \\
\hline 5 & 0 & 1 & 0 & 0 & OA'OO & 0100 & 4 \\
\hline 6 & 0 & 1 & 0 & 1 & OAION & 0101 & 5 \\
\hline 7 & 0 & 1 & 1 & 0 & $O A^{\prime} A^{\circ} O$ & 0110 & 6 \\
\hline 8 & 0 & 1 & 1 & 1 & $\circ A^{i} A^{\circ} N$ & 0111 & 7 \\
\hline 9 & 1 & 0 & 0 & 0 & Mooo & 1000 & 8 \\
\hline 10 & 1 & 0 & 0 & 1 & MooN & 1001 & 9 \\
\hline 11 & 1 & 0 & 1 & 0 & $M \circ A^{\circ} O$ & 1010 & 10 \\
\hline 12 & 1 & 0 & 1 & 1 & $M \circ A \circ N$ & 1011 & 11 \\
\hline 13 & 1 & 1 & 0 & 0 & MA'oo & 1100 & 12 \\
\hline 14 & 1 & 1 & 0 & 1 & MAION & 1101 & 13 \\
\hline 15 & 1 & 1 & 1 & 0 & $M A^{i} A^{\circ} O$ & 1110 & 14 \\
\hline 16 & 1 & 1 & 1 & 1 & $M A^{i} A^{\circ} N$ & 1111 & 15 \\
\hline
\end{tabular}

Traditional

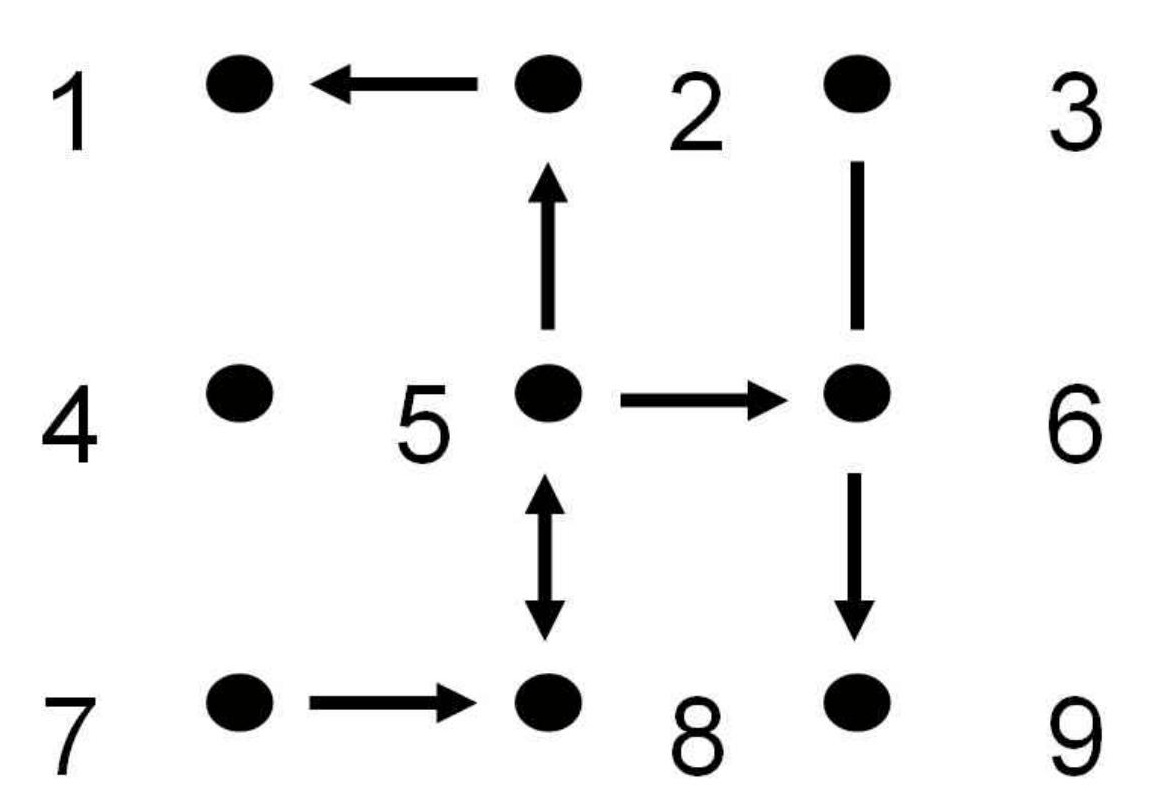

Refined Nomenclature

\section{Facilitates visualisation of complex networks}




\section{Another Step in describing complex Network}

From

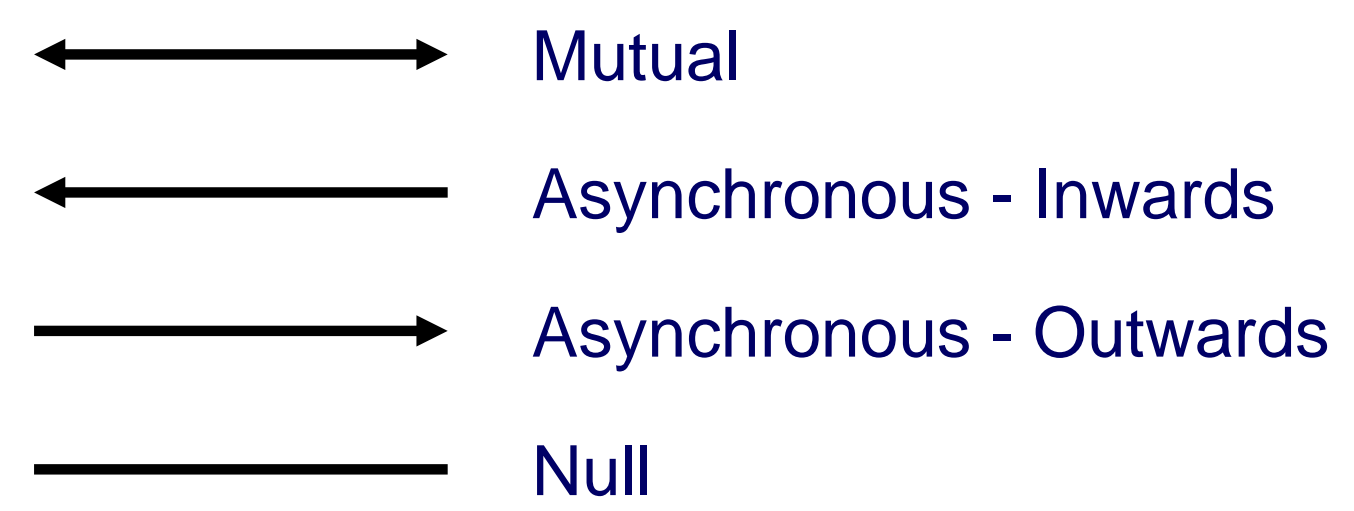

To

\section{MAN Values Table}

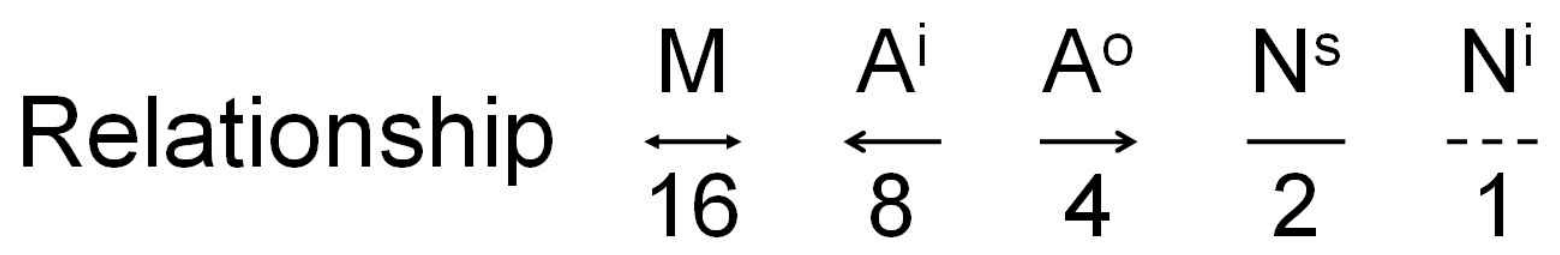


On the Matter of

Classifying Node Connections

\begin{tabular}{|c|c|c|c|c|c|c|c|c|c|c|c|c|c|c|c|c|c|c|}
\hline \multirow{4}{*}{$\begin{array}{l}\text { N } \\
\text { o } \\
\text { d } \\
\text { e } \\
\text { S }\end{array}$} & \multirow{4}{*}{$\Sigma$} & \multicolumn{5}{|c|}{ Erdos-Renvi 1 } & \multirow{4}{*}{$\Sigma$} & \multicolumn{5}{|c|}{ Erdos-Renvi 2} & \multirow{4}{*}{$\Sigma$} & \multicolumn{5}{|c|}{ Erdos-Renvi 3} \\
\hline & & \multirow{2}{*}{$\frac{\mathrm{M}}{16}$} & $\mathbf{A}^{\mathbf{i}}$ & $\mathbf{A}^{\mathbf{0}}$ & $\mathrm{N}^{s}$ & $\mathrm{~N}^{\mathrm{i}}$ & & M & $\mathbf{A}^{\mathbf{i}}$ & $\mathbf{A}^{0}$ & $\mathrm{~N}^{s}$ & $\mathrm{~N}^{\mathrm{i}}$ & & M & $\mathbf{A}^{\mathbf{i}}$ & $\mathrm{A}^{0}$ & $\mathrm{~N}^{s}$ & $\mathrm{~N}^{\mathrm{i}}$ \\
\hline & & & 8 & 4 & 2 & 1 & & 16 & 8 & 4 & 2 & 1 & & 16 & 8 & 4 & 2 & 1 \\
\hline & & \multicolumn{5}{|c|}{ Node's Link Value } & & \multicolumn{5}{|c|}{ Node's Link Value } & & \multicolumn{5}{|c|}{ Node's Link Value } \\
\hline 1 & 4 & & & 4 & & & 8 & & 8 & & & & 4 & & & 4 & & \\
\hline 2 & 12 & & 8 & 4 & & & 12 & & 8 & 4 & & & 16 & 16 & & & & \\
\hline 3 & 20 & 16 & & 4 & & & 24 & 16 & 8 & & & & 12 & & 8 & 4 & & \\
\hline 4 & 8 & & 8 & & & & 4 & & & 4 & & & 8 & & 8 & & & \\
\hline 5 & 20 & 16 & & 4 & & & 28 & 16 & 8 & 4 & & & 20 & 16 & & 4 & & \\
\hline 6 & 20 & 16 & & 4 & & & 24 & 16 & 8 & & & & 16 & 16 & & & & \\
\hline 7 & 2 & & & & 2 & & 2 & & & & 2 & & 2 & & & & 2 & \\
\hline 8 & 4 & & & 4 & & & 8 & & 8 & & & & 4 & & & 4 & & \\
\hline 9 & 4 & & & 4 & & & 16 & 16 & & & & & 20 & 16 & & 4 & & \\
\hline 10 & 8 & & 8 & & & & 4 & & & 4 & & & 8 & & 8 & & & \\
\hline 11 & 12 & & 8 & 4 & & & 8 & & 8 & & & & 12 & & 8 & 4 & & \\
\hline 12 & 4 & & & 4 & & & 4 & & & 4 & & & 4 & & & 4 & & \\
\hline 13 & 8 & & & 8 & & & 16 & 16 & & & & & 8 & & 8 & & & \\
\hline 14 & 0 & & & & & & 4 & & & 4 & & & 16 & 16 & & & & \\
\hline 15 & 8 & & 8 & & & & 16 & 16 & & & & & 8 & & 8 & & & \\
\hline 16 & 4 & & & 4 & & & 16 & 16 & & & & & 4 & & & 4 & & \\
\hline 17 & 8 & & 8 & & & & 4 & & & 4 & & & 16 & 16 & & & & \\
\hline 18 & 2 & & & & 2 & & 2 & & & & 2 & & 2 & & & & 2 & \\
\hline 19 & 24 & 16 & & 8 & & & 8 & & 8 & & & & 24 & 16 & 8 & & & \\
\hline 20 & 8 & & 8 & & & & 16 & 16 & & & & & 8 & & 8 & & & \\
\hline 21 & 2 & & & & 2 & & 2 & & & & 2 & & 2 & & & & 2 & \\
\hline 22 & 4 & & & 4 & & & 16 & 16 & & & & & 4 & & & 4 & & \\
\hline 23 & 24 & 16 & 8 & & & & 4 & & & 4 & & & 12 & & 8 & 4 & & \\
\hline 24 & 8 & & 8 & & & & 16 & 16 & & & & & 16 & 16 & & & & \\
\hline 25 & 8 & & & 8 & & & 16 & 16 & & & & & 8 & & 8 & & & \\
\hline
\end{tabular}

This phase looks at

each node individually to classify it according to the number and type of links it possesses.
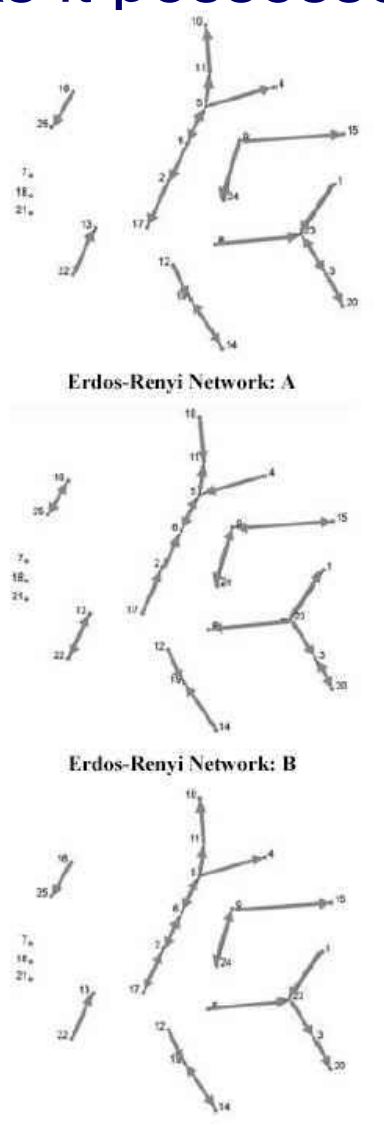

Erdos-Renyi Network: C

But, still falling short... 


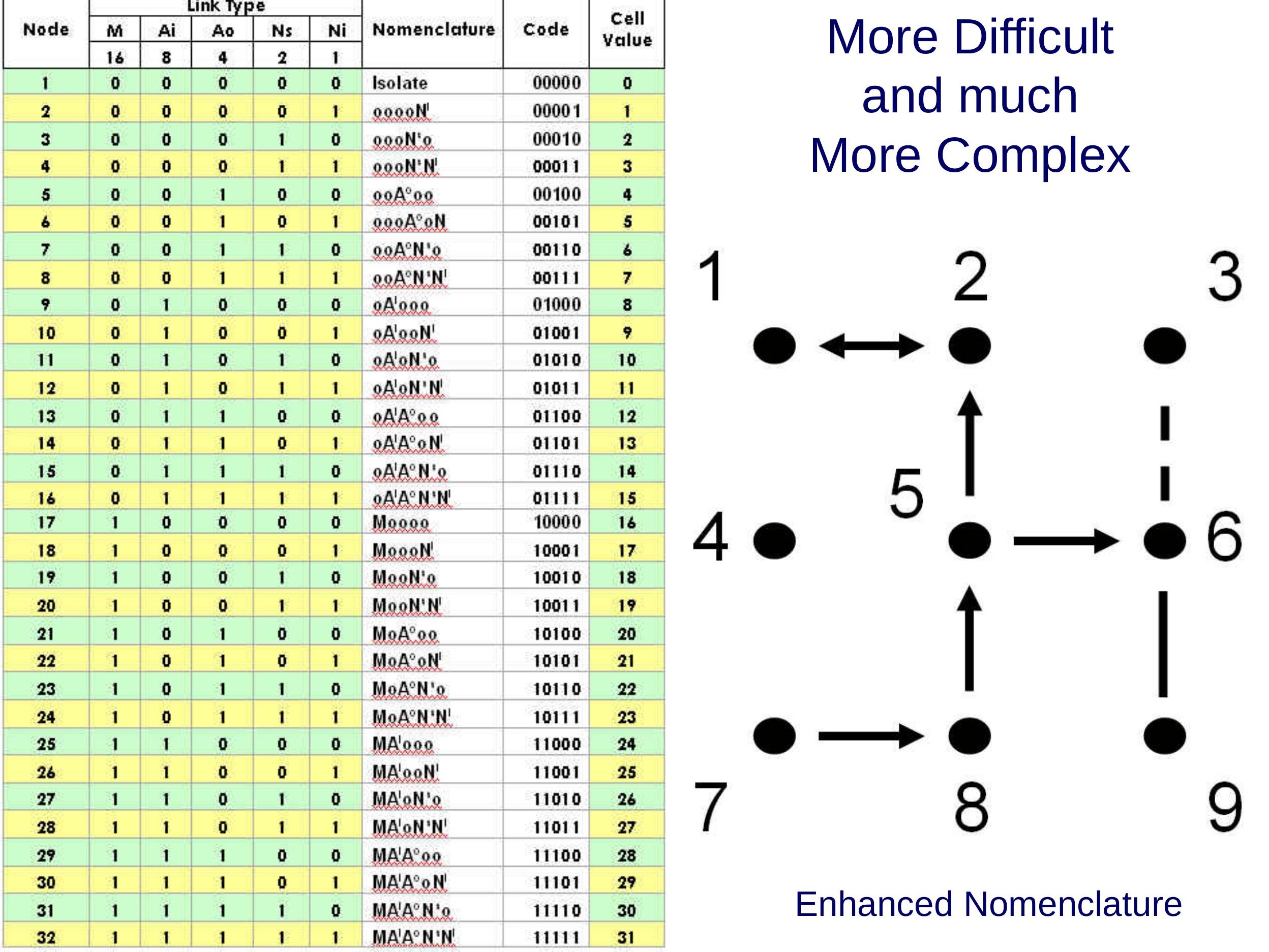




\section{A Node's 32 basic possible patterns of Relationships}

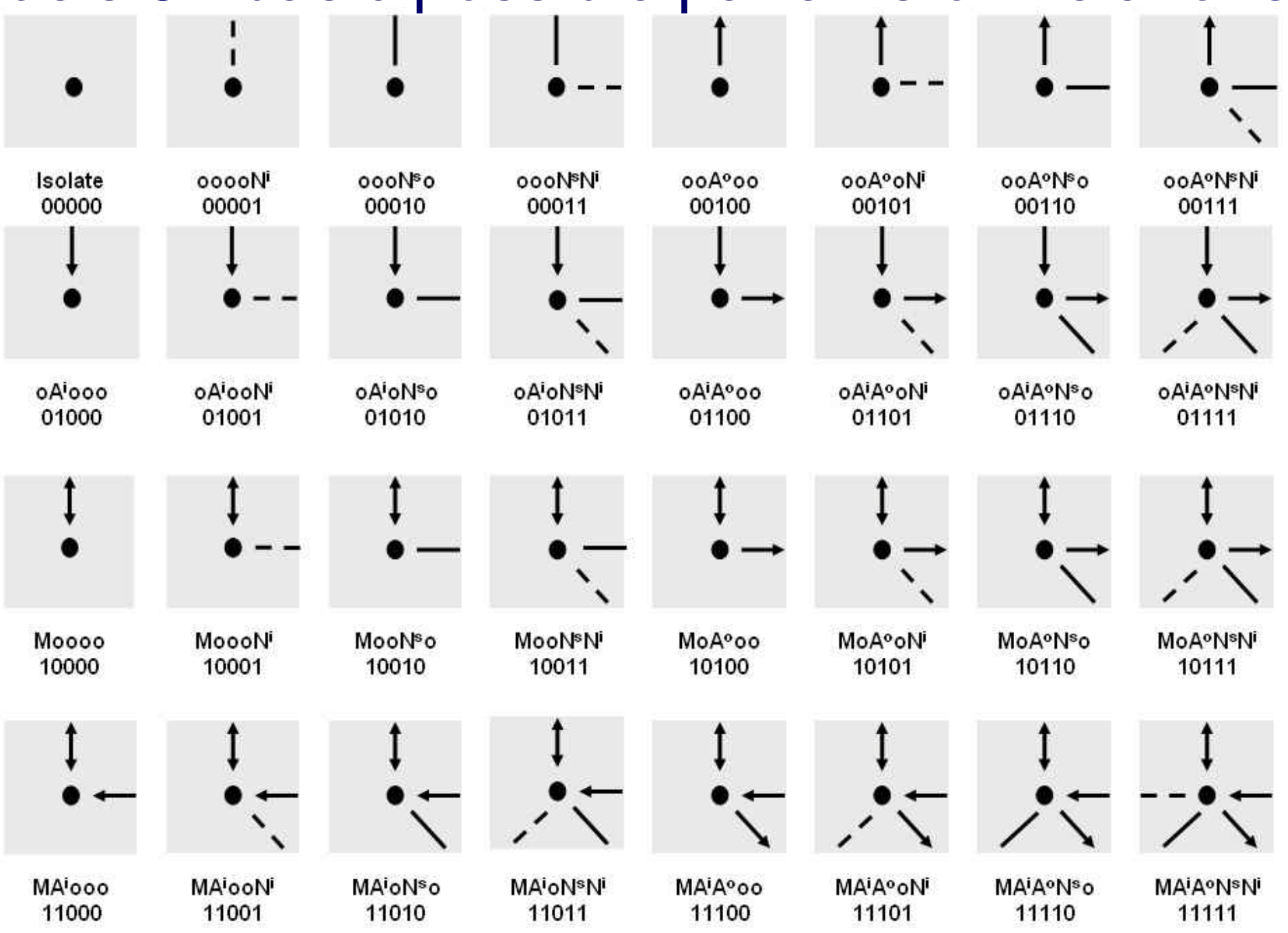

Next, managing and signify the ratio of each link type a Node in complex networks might possess 
Starting to unravel due to the limitations of $\boldsymbol{m y}$ mathematics knowledge.

\begin{tabular}{|c|c|c|c|c|c|c|c|}
\hline & MAN Extended & M & $A^{i}$ & $A^{0}$ & $\mathrm{~N}^{\mathrm{s}}$ & $\mathrm{N}^{\mathrm{i}}$ & \\
\hline & MAN Binary & 16 & 8 & 4 & 2 & 1 & \\
\hline \multirow{5}{*}{1} & $\mathrm{~N}^{0}$ of Node Links & 25 & 40 & 30 & 20 & 13 & 128 \\
\hline & Node Link Proportion & $19.53 \%$ & $31.25 \%$ & $23.44 \%$ & $15.63 \%$ & $10.15 \%$ & $100.00 \%$ \\
\hline & Proportion Classification & 2 & 3 & 2 & 2 & 1 & 10 \\
\hline & Node Link Classification & $\mathbf{M}_{2}$ & $\mathrm{~A}_{3}$ & $\mathrm{~A}^{0}{ }_{2}$ & $\mathrm{Ns}_{2}$ & $\mathrm{Ni}_{1}$ & \\
\hline & Cell Value & 32 & 24 & 4 & 4 & 1 & 65 \\
\hline \multirow{5}{*}{2} & $\mathrm{~N}^{0}$ of Node Links & 2 & 7 & 3 & 2 & 0 & 14 \\
\hline & Node Link Proportion & $19.53 \%$ & $31.25 \%$ & $23.44 \%$ & $15.63 \%$ & $10.15 \%$ & $100.00 \%$ \\
\hline & Proportion Classification & 2 & 3 & 2 & 2 & 1 & 10 \\
\hline & Node Link Classification & $\mathbf{M}_{2}$ & $\mathrm{~A}_{3} \mathbf{i}_{3}$ & $\mathrm{~A}^{0}{ }_{2}$ & $\mathrm{Ns}_{2}$ & $\mathrm{Ni}_{1}$ & \multirow{2}{*}{65} \\
\hline & Cell Value & 32 & 24 & 4 & 4 & 1 & \\
\hline \multirow{5}{*}{3} & $\mathrm{~N}^{0}$ of Node Links & 57 & 21 & 33 & 89 & 15 & 215 \\
\hline & Node Link Proportion & $26.50 \%$ & $9.77 \%$ & $15.35 \%$ & $41.40 \%$ & $6.98 \%$ & $100.00 \%$ \\
\hline & Proportion Classification & 3 & 1 & 1 & 4 & 1 & 10 \\
\hline & Node Link Classification & $\mathbf{M}_{3}$ & $\mathbf{A}_{1}^{\mathbf{i}}$ & $\mathrm{A}^{0}{ }_{1}$ & $\mathrm{Ns}_{4}$ & $\mathrm{Ni}_{1}$ & \multirow{2}{*}{69} \\
\hline & Cell Value & 48 & 8 & 4 & 8 & 1 & \\
\hline \multirow{5}{*}{4} & $\mathrm{~N}^{0}$ of Node Links & 193 & 79 & 61 & 5 & 7 & 345 \\
\hline & Node Link Proportion & $55.94 \%$ & $22.90 \%$ & $17.68 \%$ & $1.45 \%$ & $2.03 \%$ & $100.00 \%$ \\
\hline & Proportion Classification & 6 & 2 & 2 & 0 & 0 & 10 \\
\hline & Node Link Classification & $\mathbf{M}_{6}$ & $\mathrm{~A}_{2}^{\mathbf{i}_{2}}$ & $\mathrm{~A}^{0}{ }_{2}$ & $\mathrm{Ns}_{0}$ & $\mathrm{Ni}_{0}$ & \multirow{2}{*}{120} \\
\hline & Cell Value & 96 & 16 & 8 & 0 & 0 & \\
\hline \multirow{5}{*}{5} & $\mathrm{~N}^{0}$ of Node Links & 11873 & 23589 & 27156 & 1025 & 25 & 63668 \\
\hline & Node Link Proportion & $18.65 \%$ & $37.05 \%$ & $42.65 \%$ & $1.61 \%$ & $0.04 \%$ & $100.00 \%$ \\
\hline & Proportion Classification & 2 & 4 & 4 & 0 & 0 & 10 \\
\hline & Node Link Classification & $\mathbf{M}_{2}$ & $\mathrm{~A}_{4}{ }_{4}$ & $\mathrm{~A}^{0}{ }_{4}$ & $\mathrm{Ns}_{0}$ & $\mathrm{Ni}_{0}$ & \multirow{2}{*}{80} \\
\hline & Cell Value & 32 & 32 & 16 & 0 & 0 & \\
\hline
\end{tabular}

Suspect that the devil is in the detail...

What is the significance of a Node classification $\mathrm{M}_{2} \mathrm{~A}_{3} \mathrm{~A}_{2}{ }_{2} \mathrm{~N}_{2} \mathrm{Ni}_{1}$ compared with $\mathrm{M}_{2} \mathrm{~A}_{4} \mathrm{~A}^{\mathrm{O}}{ }_{4} \mathrm{~N}_{0}^{\mathrm{s}} \mathrm{N}_{0}$ In this example Nodes 1. \& 2. vary with 128 links compared with 14 yet possess the same classification nomenclature... 
My question to you is...

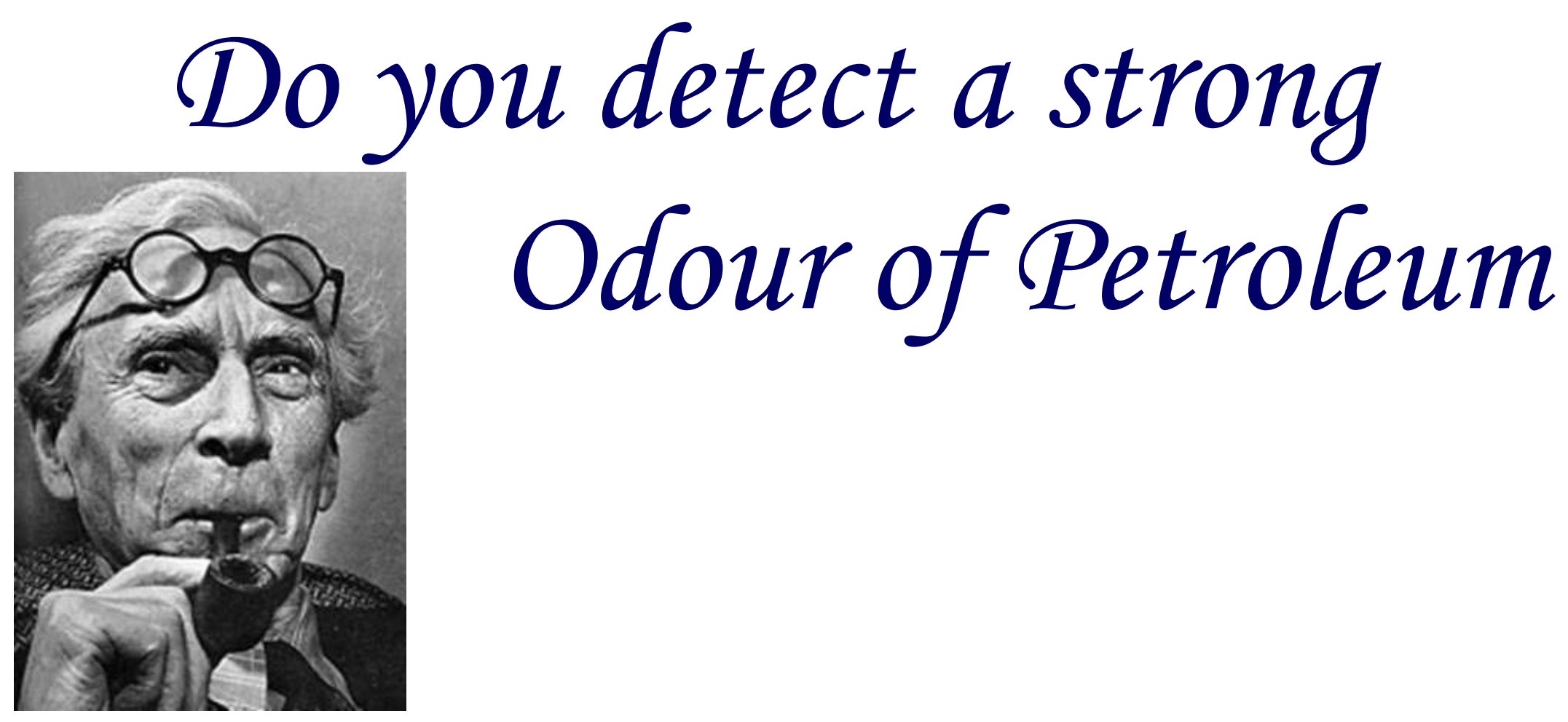



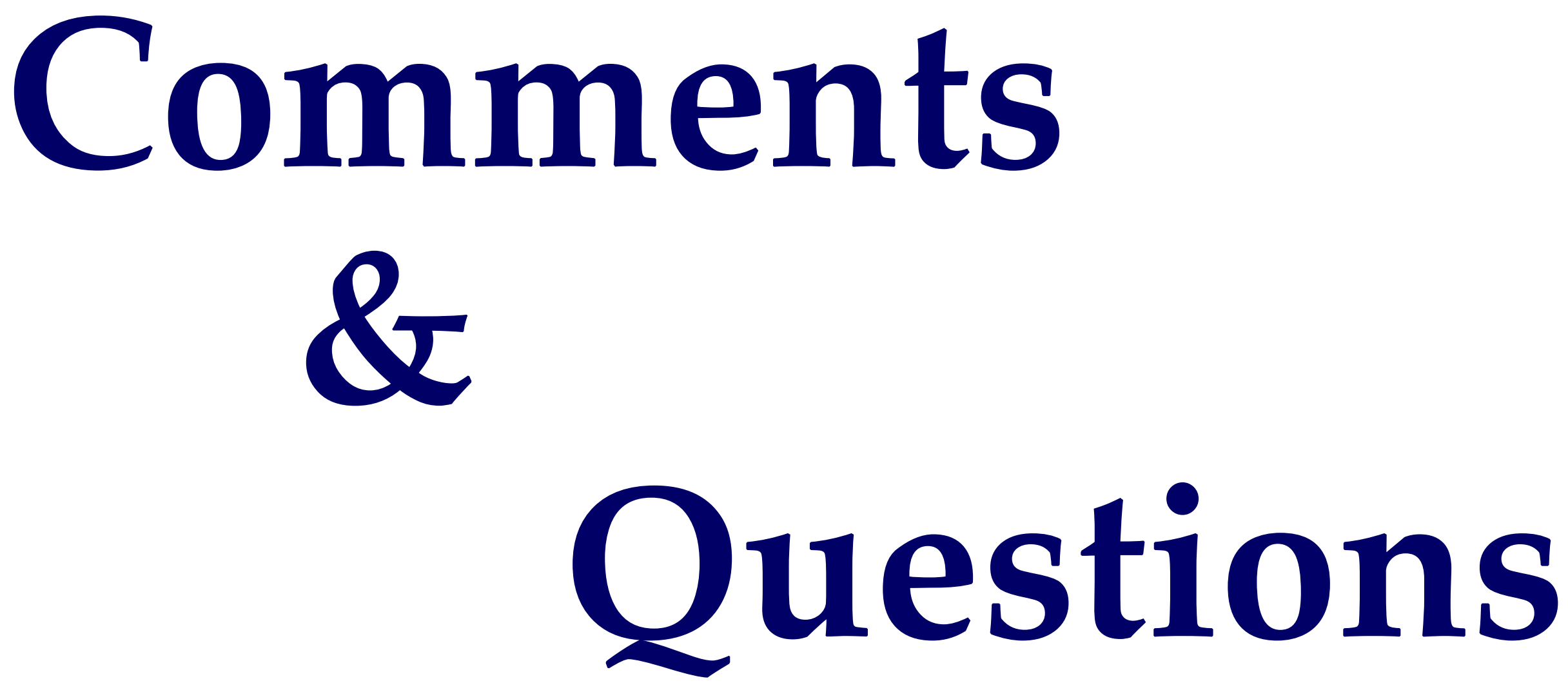

Colin J. Armstrong Curtin University Western Australia

colin.armstrong@cbs.curtin.edu.au 